Article

\title{
Existence of Small-Energy Solutions to Nonlocal Schrödinger-Type Equations for Integrodifferential Operators in $\mathbb{R}^{N}$
}

\author{
Jun Ik Lee ${ }^{1}$, Yun-Ho Kim ${ }^{1, *}$ and Jongrak Lee ${ }^{2}$ \\ 1 Department of Mathematics Education, Sangmyung University, Seoul 03016, Korea; jilee@smu.ac.kr \\ 2 Institute of Mathematical Sciences, Ewha Womans University, Seoul 120-750, Korea; jrlee0124@ewha.ac.kr \\ * Correspondence: kyh1213@smu.ac.kr; Tel.: +82-2-781-7504; Fax: +82-222870069
}

Received: 2 December 2019; Accepted: 15 December 2019; Published: 18 December 2019

check for updates

\begin{abstract}
We are concerned with the following elliptic equations: $(-\Delta)_{p, \mathcal{K}}^{s} u+V(x)|u|^{p-2} u=$ $\lambda f(x, u)$ in $\mathbb{R}^{N}$, where $(-\Delta)_{p, \mathcal{K}}^{s}$ is the nonlocal integrodifferential equation with $0<s<1<p<$ $+\infty, s p<N$ the potential function $V: \mathbb{R}^{N} \rightarrow(0, \infty)$ is continuous, and $f: \mathbb{R}^{N} \times \mathbb{R} \rightarrow \mathbb{R}$ satisfies a Carathéodory condition. The present paper is devoted to the study of the $L^{\infty}$-bound of solutions to the above problem by employing De Giorgi's iteration method and the localization method. Using this, we provide a sequence of infinitely many small-energy solutions whose $L^{\infty}$-norms converge to zero. The main tools were the modified functional method and the dual version of the fountain theorem, which is a generalization of the symmetric mountain-pass theorem.
\end{abstract}

Keywords: non-local integrodifferential operators; De Giorgi iteration; modified functional methods; dual fountain theorem

MSC: 35A15; 35J60; 35R11; 47G20

\section{Introduction}

In recent years, the study of fractional and nonlocal problems of the elliptic type has received enormous attention because the interest in such operators has sustainedly increased within the framework of mathematical theory to confirm some phenomena such as fractional quantum mechanics, material sciences, continuum mechanics, phase-transition phenomena, image processes, the thin-obstacle problem, game theory, and Lévy processes (see [1-7] and the references therein). The fractional Schrödinger equation, which was introduced by Laskin [5], has especially received considerable attention in recent years (see, e.g., [8-10]).

Stimulated by the large interest in the current literature and taking advantage of variational methods, we investigate the existence of weak solutions for nonlocal equations involving the fractional $p$-Laplacian. We establish the existence, multiplicity, and uniform estimates of infinitely many nontrivial weak solutions for the following nonlocal integrodifferential equations:

$$
(-\Delta)_{p, \mathcal{K}^{u}}^{\mathcal{u}}+V(x)|u|^{p-2} u=\lambda f(x, u) \text { in } \mathbb{R}^{N},
$$

where $\lambda$ is real parameter $0<s<1<p<+\infty$, sp $<N$, the potential function $V: \mathbb{R}^{N} \rightarrow(0, \infty)$ is continuous, $f: \mathbb{R}^{N} \times \mathbb{R} \rightarrow \mathbb{R}$ satisfies a Carathéodory condition, and $(-\Delta)_{p, \mathcal{K}}^{s}$ is the fractional $p$-Laplacian operator defined as

$$
(-\Delta)_{p, \mathcal{K}}^{s} u(x)=2 \lim _{\varepsilon \searrow 0} \int_{\mathbb{R}^{N} \backslash B_{\varepsilon}^{N}(x)}|u(x)-u(y)|^{p-2}(u(x)-u(y)) \mathcal{K}(x-y) d y
$$


for $x \in \mathbb{R}^{N}$, where $\mathcal{K}$ is a kernel function, and $B_{\mathcal{\varepsilon}}^{N}(x):=\left\{y \in \mathbb{R}^{N}:|y-x| \leq \varepsilon\right\}$. Many authors have researched fractional $p$-Laplacian type problems in various respects (see [2,3,10-19] and references therein).

The first goal of the present paper is to provide a sufficient condition ensuring global uniform boundedness for weak solutions of the problem in Equation (1). The main tools for obtaining this result are De Giorgi's iteration method and a truncated energy technique (by also considering some novel optimization techniques proposed in $[20,21])$. This approach originally comes from Vergara [22]. Inspired by Vergara [22], the boundedness of weak solutions for elliptic equations with variable exponents and a nonlinear conormal derivative boundary condition was investigated by Winkert [23] (see also [24]). The second goal is to obtain the existence of a sequence of infinitely many small-energy solutions that converge to zero in $L^{\infty}$ space. The strategy of the proof for this assertion is based on applications of the dual-fountain theorem that were primarily introduced by Bartsch [25] with consideration for the variational nature of the problem. The dual-fountain theorem, as a key tool, is a dual version of fountain theorem in [26], which is a generalization of the symmetric mountain-pass theorem in [27] and a powerful technique for ensuring the existence of multiple solutions to elliptic equations of the variational type. Subsequently, many researchers applied this theorem to the various problems involving $p$ - or $p(x)$-Laplacian (see $[8,9,28-36]$ and the references therein). Such a multiplicity result that utilizes the dual-fountain theorem to derive the existence of infinitely many small-energy solutions for nonlinear elliptic equations of the variational type can be found in $[9,29]$ (and the references therein). In [8], the author studied the existence of infinitely many solutions of the fractional Laplacian via the variant fountain theorems established in [37]. However, these consequences do not ensure the boundedness of solutions. We therefore show that nonlinear problems associated with an integrodifferential operator admits a sequence of infinitely many solutions whose $L^{\infty}$ norms converge to zero. The condition on nonlinear term $f(\cdot, t)$ at infinity and the oddness of $f$ globally are increasingly essential in obtaining previous multiplicity results, as in $[8,9,29]$. However, we infer our conclusion when conditions on $f(\cdot, t)$ are carried out near zero; $f(\cdot, t)$ is especially odd in $t$ for a small $t$, and no conditions on $f(\cdot, t)$ exist at infinity. The initial idea for this approach came from the work of Z.-Q. Wang [38], who made use of the modified functional method and global variational formulation as the main tools in [39]. Very recently, the authors of [31] investigated the $L^{\infty}$ bound of small-energy solutions to Kirchhoff-Schrödinger-type equations involving the fractional $p$-Laplacian by applying the modified functional method and a regularity-type result inspired by the work of P. Drábek, A. Kufner, and F. Nicolosi [40] (for related works, see [24,38,41-44]). We design our consequence under a somewhat different approach than previous works. In contrast to the authors of $[24,38,41-44]$, who established the existence of such a sequence of solutions belonging to the $L^{\infty}$ space, we take into account the dual-fountain theorem in place of the global variational formulation in [39]. To the best of our knowledge, there have been no results on this approach, even in classical $p$-Laplacian problems, although we derive our main result from a well-known technique.

This paper is organized as follows. We first briefly review definitions and collect some preliminary results for the Lebesgue-Sobolev space of the fractional type. Next, we present the $L^{\infty}$ bound of solutions to the problem in Equation (1) by applying De Giorgi's iteration method and the localization method. Finally, we provide a sequence of infinitely many small-energy solutions whose $L^{\infty}$-norms converge to zero.

\section{Preliminaries}

In this section, we briefly recall definitions and some elementary properties of fractional Sobolev spaces (refer to $[4,45,46]$ for further reference). For simplicity, $\mathcal{C}$ is used to represent a generic constant that may change from line to line. 
Let $s \in(0,1), p \in(1,+\infty)$, and $p_{s}^{*}$ is the fractional critical Sobolev exponent, that is

$$
p_{s}^{*}:= \begin{cases}\frac{N p}{N-s p} & \text { if } s p<N \\ +\infty & \text { if } s p \geq N .\end{cases}
$$

We define fractional Sobolev space $W_{\mathcal{K}}^{s, p}\left(\mathbb{R}^{N}\right)$ as follows:

$$
W_{\mathcal{K}}^{s, p}\left(\mathbb{R}^{N}\right):=\left\{u \in L^{p}\left(\mathbb{R}^{N}\right): \int_{\mathbb{R}^{N}} \int_{\mathbb{R}^{N}}|u(x)-u(y)|^{p} \mathcal{K}(x-y) d x d y<+\infty\right\},
$$

where $\mathcal{K}: \mathbb{R}^{N} \backslash\{0\} \rightarrow(0,+\infty)$ is a kernel function satisfying the following properties:

(K1) $m \mathcal{K} \in L^{1}\left(\mathbb{R}^{N}\right)$, where $m(x)=\min \left\{|x|^{p}, 1\right\}$;

(K2) there exist $d_{1}, d_{2}>0$ such that $d_{1}|x|^{-(N+p s)} \leq \mathcal{K}(x) \leq d_{2}|x|^{-(N+p s)}$ for all $x \in \mathbb{R}^{N} \backslash\{0\}$;

(K3) $\mathcal{K}(x)=\mathcal{K}(-x)$ for all $x \in \mathbb{R}^{N} \backslash\{0\}$.

By Condition (K1), function

$$
(x, y) \mapsto(u(x)-u(y)) \mathcal{K}^{\frac{1}{p}}(x-y) \in L^{p}\left(\mathbb{R}^{2 N}\right)
$$

for all $u \in C_{0}^{\infty}\left(\mathbb{R}^{N}\right)$. Let us denote by $W_{\mathcal{K}}^{s, p}\left(\mathbb{R}^{N}\right)$ as the completion of $C_{0}^{\infty}\left(\mathbb{R}^{N}\right)$ with respect to norm

$$
\|u\|_{W_{\mathcal{K}}^{s, p}\left(\mathbb{R}^{N}\right)}:=\left(\|u\|_{L^{p}\left(\mathbb{R}^{N}\right)}^{p}+|u|_{W_{\mathcal{K}}^{s, p}\left(\mathbb{R}^{N}\right)}^{p}\right)^{\frac{1}{p}}
$$

where

$$
|u|_{W_{\mathcal{K}}^{s, p}\left(\mathbb{R}^{N}\right)}^{p}:=\int_{\mathbb{R}^{N}} \int_{\mathbb{R}^{N}}|u(x)-u(y)|^{p} \mathcal{K}(x-y) d x d y .
$$

Lemma 1. [47] Let $\mathcal{K}: \mathbb{R}^{N} \backslash\{0\} \rightarrow(0, \infty)$ be a kernel function satisfying Conditions (K1)-(K3). If $v \in W_{\mathcal{K}}^{s, p}\left(\mathbb{R}^{N}\right)$, then $v \in W^{s, p}\left(\mathbb{R}^{N}\right)$. Moreover,

$$
\|v\|_{W^{s, p}\left(\mathbb{R}^{N}\right)} \leq \max \left\{1, d_{1}^{-\frac{1}{p}}\right\}\|v\|_{W_{\mathcal{K}}^{s, p}\left(\mathbb{R}^{N}\right)} ;
$$

Then, $W^{s, p}\left(\mathbb{R}^{N}\right)$ is a separable and reflexive Banach space. Space $C_{0}^{\infty}\left(\mathbb{R}^{N}\right)$ is also dense in $W^{s, p}\left(\mathbb{R}^{N}\right)$, which is $W_{0}^{s, p}\left(\mathbb{R}^{N}\right)=W^{s, p}\left(\mathbb{R}^{N}\right)$ (see, e.g., [45,46]).

Lemma 2. [48] Let $\Omega \subset \mathbb{R}^{N}$ be a bounded open set with Lipschitz boundary $s \in(0,1)$ and $p \in(1,+\infty)$. Then, we have the following continuous embeddings:

$$
\begin{aligned}
& W^{s, p}(\Omega) \hookrightarrow L^{q}(\Omega) \quad \text { for all } q \in\left[1, p_{s}^{*}\right], \quad \text { if } s p<N \text {; } \\
& W^{s, p}(\Omega) \hookrightarrow L^{q}(\Omega) \quad \text { for every } q \in[1, \infty), \quad \text { if } s p=N \text {; } \\
& W^{s, p}(\Omega) \hookrightarrow C_{b}^{0, \lambda}(\Omega) \text { for all } \lambda<s-N / p, \quad \text { if } s p>N \text {. }
\end{aligned}
$$

In particular, space $W^{s, p}(\Omega)$ is compactly embedded in $L^{q}(\Omega)$ for any $q \in\left[p, p_{s}^{*}\right)$.

Lemma 3. [46,49] Let $0<s<1<p<+\infty$ with $p s<N$. Then, there exists positive constant $C=C(N, p, s)$, such that, for all $u \in W^{s, p}\left(\mathbb{R}^{N}\right)$,

$$
\|u\|_{L^{p_{s}^{*}\left(\mathbb{R}^{N}\right)}} \leq C|u|_{W^{s, p}\left(\mathbb{R}^{N}\right)} .
$$

Consequently, space $W^{s, p}\left(\mathbb{R}^{N}\right)$ is continuously embedded in $L^{q}\left(\mathbb{R}^{N}\right)$ for any $q \in\left[p, p_{s}^{*}\right]$. Moreover, embedding

$$
W^{s, p}\left(\mathbb{R}^{N}\right) \hookrightarrow L_{l o c}^{q}\left(\mathbb{R}^{N}\right)
$$


is compact for $q \in\left[p, p_{s}^{*}\right)$. In particular, we denote the best constant $S_{s, p}$ in the fractional Sobolev inequality by

$$
S_{s, p}=\inf _{u \in L^{p_{s}^{*}}\left(\mathbb{R}^{N}\right) \backslash\{0\},|u|_{W^{s, p}\left(\mathbb{R}^{N}\right)}<\infty} \frac{|u|_{W^{s, p}\left(\mathbb{R}^{N}\right)}^{p}}{\|u\|_{L^{p_{s}^{*}}\left(\mathbb{R}^{N}\right)}^{p}} .
$$

From Lemmas 1-3, we can immediately obtain the following assertion.

Lemma 4. [47] Let $\mathcal{K}: \mathbb{R}^{N} \backslash\{0\} \rightarrow(0, \infty)$ satisfy Conditions (K1)-(K3). Then, there exists positive constant $C_{0}=C_{0}(N, p, s)$, such that, for any $v \in W_{\mathcal{K}}^{s, p}\left(\mathbb{R}^{N}\right)$ and $p \leq q \leq p_{s}^{*}$,

$$
\begin{aligned}
\|v\|_{L^{q}\left(\mathbb{R}^{N}\right)}^{p} & \leq C_{0} \int_{\mathbb{R}^{N}} \int_{\mathbb{R}^{N}} \frac{|v(x)-v(y)|^{p}}{|x-y|^{N+p s}} d x d y \\
& \leq \frac{C_{0}}{d_{1}} \int_{\mathbb{R}^{N}} \int_{\mathbb{R}^{N}}|v(x)-v(y)|^{p} \mathcal{K}(x-y) d x d y .
\end{aligned}
$$

Consequently, space $W_{\mathcal{K}}^{s, p}\left(\mathbb{R}^{N}\right)$ is continuously embedded in $L^{q}\left(\mathbb{R}^{N}\right)$ for any $q \in\left[p, p_{s}^{*}\right]$. Moreover, embedding

$$
W_{\mathcal{K}}^{s, p}\left(\mathbb{R}^{N}\right) \hookrightarrow L_{l o c}^{q}\left(\mathbb{R}^{N}\right)
$$

is compact for $q \in\left[p, p_{s}^{*}\right)$.

For potential term $V$, we assume that

(V) $V \in C\left(\mathbb{R}^{N}\right), V_{-}:=\inf _{x \in \mathbb{R}^{N}} V(x)>0$, meas $\left\{x \in \mathbb{R}^{N}: V(x) \leq v_{0}\right\}<+\infty$ for all $v_{0} \in \mathbb{R}$.

On linear subspace

$$
E:=\left\{u \in W^{s, p}\left(\mathbb{R}^{N}\right): \int_{\mathbb{R}^{N}} \int_{\mathbb{R}^{N}} \frac{|u(x)-u(y)|^{p}}{|x-y|^{N+s p}} d x d y+\int_{\mathbb{R}^{N}} V(x)|u(x)|^{p} d x<+\infty\right\},
$$

we equip norm

$$
\|u\|_{E}:=\inf \left\{\lambda>0: \int_{\mathbb{R}^{N}} \int_{\mathbb{R}^{N}} \frac{|u(x)-u(y)|^{p}}{\lambda^{p}|x-y|^{N+s p}} d x d y+\int_{\mathbb{R}^{N}} V(x)\left|\frac{u(x)}{\lambda}\right|^{p} d x<1\right\} .
$$

Then, $\left(E,\|\cdot\|_{E}\right)$ is continuously embedded into $W^{s, p}\left(\mathbb{R}^{N}\right)$ as a closed subspace. Therefore, $(E, \| \cdot$ $\left.\|_{E}\right)$ is also separable reflexive Banach space.

We can then verify that $W_{\mathcal{K}, V}^{s, p}\left(\mathbb{R}^{N}\right)$ is a separable and reflexive Banach space with the following norm:

$$
\|u\|_{W_{\mathcal{K}, V}^{s, p}\left(\mathbb{R}^{N}\right)}:=\left(\|u\|_{L_{V}^{p}\left(\mathbb{R}^{N}\right)}^{p}+|u|_{W_{\mathcal{K}}^{s, p}\left(\mathbb{R}^{N}\right)}^{p}\right)^{\frac{1}{p}}
$$

where

$$
\|u\|_{L_{V}^{p}\left(\mathbb{R}^{N}\right)}^{p}:=\int_{\mathbb{R}^{N}} V(x)|u|^{p} d x .
$$

Throughout this paper, we denote $X\left(\mathbb{R}^{N}\right):=W_{\mathcal{K}, V}^{s, p}\left(\mathbb{R}^{N}\right)$, and function $\mathcal{K}: \mathbb{R}^{N} \backslash\{0\} \rightarrow(0, \infty)$ satisfies Conditions (K1)-(K3).

In view of Lemmas 2 and 4 , the following result is carried out by the same scheme as that in [50].

Lemma 5. Let $0<s<1<p<+\infty$ with $p s<N$, and suppose that Assumption (V) holds. Then, there is compact embedding $X\left(\mathbb{R}^{N}\right) \hookrightarrow L^{q}\left(\mathbb{R}^{N}\right)$ for $q \in\left[p, p_{s}^{*}\right)$. 
Definition 1. We indicate that $u \in X\left(\mathbb{R}^{N}\right)$ is a weak solution to the problem in Equation (1) if

$$
\begin{gathered}
\int_{\mathbb{R}^{N}} \int_{\mathbb{R}^{N}}|u(x)-u(y)|^{p-2}(u(x)-u(y))(v(x)-v(y)) \mathcal{K}(x-y) d x d y \\
+\int_{\mathbb{R}^{N}} V(x)|u(x)|^{p-2} u v d x=\lambda \int_{\mathbb{R}^{N}} f(x, u) v d x
\end{gathered}
$$

for any $v \in X\left(\mathbb{R}^{N}\right)$.

Let us define functional $\Phi_{s, p}: X\left(\mathbb{R}^{N}\right) \rightarrow \mathbb{R}$ by

$$
\Phi_{s, p}(u)=\frac{1}{p} \int_{\mathbb{R}^{N}} \int_{\mathbb{R}^{N}}|u(x)-u(y)|^{p} \mathcal{K}(x-y) d x d y+\frac{1}{p} \int_{\mathbb{R}^{N}} V(x)|u(x)|^{p} d x .
$$

Then, from the modification of Lemma 3.2 of [50], functional $\Phi_{s, p}$ is well-defined on $X\left(\mathbb{R}^{N}\right)$, $\Phi_{s, p} \in C^{1}\left(X\left(\mathbb{R}^{N}\right), \mathbb{R}\right)$ and, for any $v \in X\left(\mathbb{R}^{N}\right)$, its Fréchet derivative is given by

$$
\begin{gathered}
\left\langle\Phi_{s, p}^{\prime}(u), v\right\rangle=\int_{\mathbb{R}^{N}} \int_{\mathbb{R}^{N}}|u(x)-u(y)|^{p-2}(u(x)-u(y))(v(x)-v(y)) \mathcal{K}(x-y) d x d y \\
+\int_{\mathbb{R}^{N}} V(x)|u(x)|^{p-2} u v d x .
\end{gathered}
$$

For $p<q<p_{s}^{*}$, we assume that

(F1) $f: \mathbb{R}^{N} \times \mathbb{R} \rightarrow \mathbb{R}$ satisfies the Carathéodory condition.

(F2) There exist non-negative functions $\rho \in L^{p^{\prime}}\left(\mathbb{R}^{N}\right) \cap L^{\infty}\left(\mathbb{R}^{N}\right)$ and $\sigma \in L^{\frac{p_{s}^{*}}{p_{s}^{*}-q}}\left(\mathbb{R}^{N}\right) \cap L^{\infty}\left(\mathbb{R}^{N}\right)$ such that

$$
|f(x, t)| \leq \rho(x)+\sigma(x)|t|^{q-1}
$$

for all $(x, t) \in \mathbb{R}^{N} \times \mathbb{R}$.

Under Assumptions (F1) and (F2), we define functional $\Psi: X\left(\mathbb{R}^{N}\right) \rightarrow \mathbb{R}$ by

$$
\Psi(u)=\int_{\mathbb{R}^{N}} F(x, u) d x .
$$

Then, it follows from the same arguments as those of Proposition 1.12 in [51] that $\Psi \in C^{1}\left(X\left(\mathbb{R}^{N}\right), \mathbb{R}\right)$, and, for any $u, v \in X\left(\mathbb{R}^{N}\right)$, its Fréchet derivative is

$$
\left\langle\Psi^{\prime}(u), v\right\rangle=\int_{\mathbb{R}^{N}} f(x, u) v d x .
$$

Next, functional $I_{\lambda}: X\left(\mathbb{R}^{N}\right) \rightarrow \mathbb{R}$ is defined by

$$
I_{\lambda}(u)=\Phi_{s, p}(u)-\lambda \Psi(u) .
$$

Then, we know that $I_{\lambda} \in C^{1}\left(X\left(\mathbb{R}^{N}\right), \mathbb{R}\right)$, and the Fréchet derivative of $I_{\lambda}$ is

$$
\begin{gathered}
\left\langle I_{\lambda}^{\prime}(u), v\right\rangle=\int_{\mathbb{R}^{N}} \int_{\mathbb{R}^{N}}|u(x)-u(y)|^{p-2}(u(x)-u(y))(v(x)-v(y)) \mathcal{K}(x-y) d x d y \\
\quad+\int_{\mathbb{R}^{N}} V(x)|u(x)|^{p-2} u v d x-\lambda \int_{\mathbb{R}^{N}} f(x, u) v d x
\end{gathered}
$$

for any $u, v \in X\left(\mathbb{R}^{N}\right)$. 


\section{Main Result}

In this section, we present the $L^{\infty}$ bound of solutions to the problem in Equation (1) and then establish the existence of a sequence of infinitely many small-energy solutions whose $L^{\infty}$ norms converge to zero by employing the modified functional method and the dual-fountain theorem.

To utilize the De Giorgi technique, we needed the following vital lemma. The proof is in ([22], Lemma 2.2).

Lemma 6. Let $\left\{Z_{n}\right\}_{n=1}^{\infty}$ be a sequence of positive numbers satisfying recursion inequality

$$
Z_{n+1} \leq c b^{n} Z_{n}^{1+\delta}, \quad n=0,1,2, \cdots
$$

for some $b>1, c>0$ and $\delta>0$. If $Z_{0} \leq \min \left\{1, c^{(-1) / \delta} b^{(-1) / \delta^{2}}\right\}$, then $Z_{n} \leq 1$ for some $n \in \mathbb{N} \cup\{0\}$. Moreover,

$$
Z_{n} \leq \min \left\{1, c^{(-1) / \delta} b^{(-1) / \delta^{2}} b^{(-n) / \delta}\right\}
$$

for any $n \geq n_{0}$, where $n_{0}$ is the smallest $n \in \mathbb{N} \cup\{0\}$ satisfying $Z_{n} \leq 1$. In particular, $Z_{n} \rightarrow 0$ as $n \rightarrow \infty$.

For convenience, we define the fractional $(s, p)$ gradient of function $v \in X\left(\mathbb{R}^{N}\right)$ as

$$
\left|D^{s} v(x)\right|^{p}=\int_{\mathbb{R}^{N}}|v(x+h)-v(x)|^{p} \mathcal{K}(h) d h .
$$

This $(s, p)$ gradient is well-defined in $\mathbb{R}^{N}$ and $\left|D^{s} v\right| \in L^{p}\left(\mathbb{R}^{N}\right)$ (see [52]).

Next, we show the following assertion, which is a regularity-type result via the De Giorgi technique and the localization method.

Proposition 1. Assume that Assumptions (V) and (F1)-(F2) hold. If $u$ is a weak solution of Problem $\left(P_{\lambda}\right)$, then $u \in L^{\infty}\left(\mathbb{R}^{N}\right)$, and there exists positive constant $\tau$ that is independent of $u$, such that

$$
\|u\|_{L^{\infty}\left(\mathbb{R}^{N}\right)} \leq \mathcal{C}\|u\|_{L^{q}\left(\mathbb{R}^{N}\right)}^{\tau}
$$

Proof. Let $A_{k}=\left\{x \in \mathbb{R}^{N}: u(x)>k\right\}, \tilde{A_{k}}=\left\{x \in \mathbb{R}^{N}:-u(x)>k\right\}$ for $k>0 .\left|A_{k}\right|$ and $\left|\tilde{A_{k}}\right|$ are finite for any $k \in \mathbb{N}$. Taking test function $v=(u-k)_{+} \in X\left(\mathbb{R}^{N}\right)$ in the problem in Equation (1) and integrating it over $\mathbb{R}^{N}$, we have

$$
\begin{gathered}
\int_{\mathbb{R}^{N}} \int_{\mathbb{R}^{N}}|u(x)-u(y)|^{p-2}(u(x)-u(y))(v(x)-v(y)) \mathcal{K}(x-y) d x d y \\
+\int_{\mathbb{R}^{N}} V(x)|u(x)|^{p-2} u v d x=\lambda \int_{\mathbb{R}^{N}} f(x, u) v d x
\end{gathered}
$$

Equivalently,

$$
\int_{A_{k}} \int_{A_{k}}|u(x)-u(y)|^{p} \mathcal{K}(x-y) d x d y+\int_{A_{k}} V(x)|u(x)|^{p-2} u(u-k) d x \leq \lambda \int_{A_{k}} f(x, u)(u-k) d x .
$$

Hence, since $u \geq u-k>0$ on $A_{k}$, by Assumption (F2),

$$
\begin{aligned}
\int_{A_{k}}\left|D^{s} u\right|^{p} d x & \leq-\int_{A_{k}} V(x)|u|^{p-2} u(u-k) d x+\lambda \int_{A_{k}}\left(\rho(x)+\sigma(x)|u|^{q-1}\right)(u-k) d x \\
& \leq \lambda \int_{A_{k}}\left(\rho(x)+\sigma(x)|u|^{q-1}\right) u d x \\
& \leq \lambda\|\rho\|_{L^{\infty}\left(\mathbb{R}^{N}\right)} \int_{A_{k}} u d x+\lambda\|\sigma\|_{L^{\infty}\left(\mathbb{R}^{N}\right)} \int_{A_{k}} u^{q} d x
\end{aligned}
$$




$$
\leq \lambda\left(1+k_{*}^{1-q}\right)\left(\|\rho\|_{L^{\infty}\left(\mathbb{R}^{N}\right)}+\|\sigma\|_{L^{\infty}\left(\mathbb{R}^{N}\right)}\right) \int_{A_{k}} u^{q} d x
$$

where $k_{*}>0$, specified below. Put $k_{n}:=k_{*}\left(2-1 / 2^{n}\right), n=0,1,2, \cdots$ and

$$
Z_{n}:=\int_{A_{k_{n}}}\left(u-k_{n}\right)^{q} d x .
$$

Since $k_{*} \leq k_{n} \leq k_{n+1} \leq 2 k_{*}$ for all $n \in \mathbb{N}$, we have

$$
\int_{A_{k_{n}}}\left(u-k_{n}\right)^{q} d x \geq \int_{A_{k_{n+1}}} u^{q}\left(1-\frac{k_{n}}{k_{n+1}}\right)^{q} d x \geq \int_{A_{k_{n+1}}} \frac{u^{q}}{2^{q(n+2)}} d x
$$

and

$$
Z_{n} \geq \int_{A_{k_{n+1}}} \frac{u^{q}}{2^{q(n+2)}} d x
$$

Thus,

$$
\int_{A_{k_{n+1}}} u^{q} d x \leq e_{1}^{n+2} Z_{n}
$$

where $e_{1}=2^{q}>1$. It follows from the relationships in Equations (3) and (4) that

$$
\int_{A_{k_{n+1}}}\left|D^{s}\left(u-k_{n+1}\right)\right|^{p} d x \leq \lambda\left(1+k_{*}^{1-q}\right)\left(\|\rho\|_{L^{\infty}\left(\mathbb{R}^{N}\right)}+\|\sigma\|_{L^{\infty}\left(\mathbb{R}^{N}\right)}\right) e_{1}^{n+2} Z_{n}
$$

For the Lebesgue measure of $A_{k_{n+1}}$, we deduced that

$$
\left|A_{k_{n+1}}\right| \leq \int_{A_{k_{n+1}}}\left(\frac{u-k_{n}}{k_{n+1}-k_{n}}\right)^{q} d x=\int_{A_{k_{n+1}}}\left(\frac{2^{n+1}}{k_{*}}\right)^{q}\left(u-k_{n}\right)^{q} d x
$$

Thus,

$$
\left|A_{k_{n+1}}\right| \leq \frac{e_{1}^{n+1}}{k_{*}^{q}} Z_{n}
$$

Then,

$$
\int_{A_{k_{n+1}}}\left|D^{s}\left(u-k_{n+1}\right)\right|^{p} d x \geq \int_{A_{k_{n+1}}}\left|D^{s}\left(u-k_{n+1}\right)\right|^{p} d x-\left|A_{k_{n+1}}\right| .
$$

Therefore, it follows from the above inequality and the relations in Equations (5) and (6) that

$$
\begin{aligned}
\int_{A_{k_{n+1}}}\left|D^{s}\left(u-k_{n+1}\right)\right|^{p} d x & \leq \lambda\left(1+k_{*}^{1-q}\right)\left(\|\rho\|_{L^{\infty}\left(\mathbb{R}^{N}\right)}+\|\sigma\|_{L^{\infty}\left(\mathbb{R}^{N}\right)}\right) e_{1}^{n+2} Z_{n}+\left|A_{k_{n+1}}\right| \\
& \leq \lambda\left(1+k_{*}^{1-q}\right)\left(\|\rho\|_{L^{\infty}\left(\mathbb{R}^{N}\right)}+\|\sigma\|_{L^{\infty}\left(\mathbb{R}^{N}\right)}\right) e_{1}^{n+2} Z_{n}+\frac{e_{1}^{n+1}}{k_{*}^{q}} Z_{n} \\
& =e_{2} e_{1}^{n} Z_{n}
\end{aligned}
$$

where $e_{2}:=\lambda\left(1+k_{*}^{1-q}\right)\left(\|\rho\|_{L^{\infty}\left(\mathbb{R}^{N}\right)}+\|\sigma\|_{L^{\infty}\left(\mathbb{R}^{N}\right)}\right) e_{1}^{2}+\frac{e_{1}}{k_{*}^{q}}$. Define

$$
\tilde{q}:= \begin{cases}\frac{q+p_{s}^{*}}{2} & \text { if } p_{s}^{*}<\infty \\ q+1 & \text { if } p_{s}^{*}=\infty\end{cases}
$$

Using the Hölder inequality and Lemma 3, we get 


$$
\begin{aligned}
\int_{A_{k_{n+1}}} & \left(u-k_{n+1}\right)_{+}^{q} d x \\
\leq & \left(\int_{\mathbb{R}^{N}}\left(u-k_{n+1}\right)_{+}^{\tilde{q}} d x\right)^{q / \tilde{q}}\left|A_{k_{n+1}}\right|^{1-q / \tilde{q}} \\
\leq & \left\|\left(u-k_{n+1}\right)_{+}\right\|_{L^{\tilde{q}\left(\mathbb{R}^{N}\right)}}^{q}\left|A_{k_{n+1}}\right|^{1-q / \tilde{q}} \\
& \leq \mathcal{C}^{q}\left(\left\|\left(u-k_{n+1}\right)_{+}\right\|_{L^{p}\left(\mathbb{R}^{N}\right)}^{p}+\left|\left(u-k_{n+1}\right)_{+}\right|_{W_{\mathcal{K}}^{s, p}\left(\mathbb{R}^{N}\right)}^{p}\right)^{q / p}\left|A_{k_{n+1}}\right|^{1-q / \tilde{q}} .
\end{aligned}
$$

Meanwhile, we have

$$
\int_{\mathbb{R}^{N}}\left|\left(u-k_{n+1}\right)_{+}\right|^{p} d x \leq \int_{A_{k_{n+1}}} u^{q} d x \leq e_{1}^{n+2} Z_{n}
$$

from Equation (7), we estimate

$$
\int_{\mathbb{R}^{N}}\left|D^{s}\left(u-k_{n+1}\right)_{+}\right|^{p} d x \leq \int_{A_{k_{n+1}}}\left|D^{s}\left(u-k_{n+1}\right)\right|^{p} d x \leq e_{2} e_{1}^{n} Z_{n} .
$$

We deduce from Equations (6), (8), and (9) that

$$
\begin{aligned}
Z_{n+1} & =\int_{A_{k_{n+1}}}\left(u-k_{n+1}\right)^{q} d x \\
& \leq \mathcal{C}^{q}\left(e_{1}^{n+2} Z_{n}+e_{2} e_{1}^{n} Z_{n}\right)^{q / p}\left(\frac{e_{1}^{n+1}}{k_{*}^{q}}\right)^{1-q / \tilde{q}} Z_{n}^{1-q / \tilde{q}} \\
& =\mathcal{C}^{q}\left(e_{1}^{2}+e_{2}\right)^{q / p}\left(\frac{e_{1}}{k_{*}^{q}}\right)^{1-q / \tilde{q}} e_{1}^{(1-q / \tilde{q}+q / p) n} Z_{n}^{1-q / \tilde{q}+q / p} .
\end{aligned}
$$

In other words,

$$
Z_{n+1} \leq \frac{e_{3}}{k_{*}^{q(1-q / \tilde{q})}} e_{1}^{(1+\delta) n} Z_{n}^{1+\delta},
$$

where $e_{3}=\mathcal{C}^{q}\left(e_{1}^{2}+e_{2}\right)^{q / p}\left(e_{1}\right)^{1-q / \tilde{q}}$ and $\delta=q / p-q / \tilde{q}$. Applying Lemma 6 with Equation (10), we obtain that

$$
Z_{n}=\int_{\mathbb{R}^{N}}\left(u-k_{n}\right)_{+}^{q} d x \rightarrow 0 \text { as } n \rightarrow \infty,
$$

provided that

$$
Z_{0} \leq \min \left\{1,\left(\frac{e_{3}}{k_{*}^{q\left(1-\frac{q}{q}\right)}}\right)^{-\frac{1}{\delta}} e_{1}^{-\frac{1+\delta}{\delta^{2}}}\right\}
$$

Observe that

$$
Z_{0}=\int_{A_{k *}}\left(u-k_{*}\right)^{q} d x \leq \int_{\mathbb{R}^{N}} u_{+}^{q} d x
$$

Set

$$
k_{*}=\left(e_{3}\right)^{\frac{\tilde{q}}{q(\tilde{q}-q)}} e_{1}^{\left(\frac{\tilde{q}}{q(\tilde{q}-q)}\right)\left(1+\frac{1}{\delta}\right)}\left(\int_{\mathbb{R}^{N}} u_{+}^{q} d x\right)^{\frac{\delta \tilde{q}}{q(\tilde{q}-q)}} .
$$


Thus, we have inequality $Z_{0} \leq 1$ if $\int_{\mathbb{R}^{N}} u_{+}^{q} d x \leq 1$ or inequality $Z_{0} \leq\left(\frac{e_{3}}{k_{*}^{q\left(1-\frac{q}{q}\right)}}\right)^{-\frac{1}{\delta}} e_{1}^{-\frac{1+\delta}{\delta^{2}}}$ if $\int_{\mathbb{R}^{N}} u_{+}^{q} d x>1$. Since $k_{n} \uparrow 2 k_{*}$, the relation in Equation (11) implies that

$$
\int_{\mathbb{R}^{N}}\left(u-2 k_{*}\right)_{+}^{q} d x=0
$$

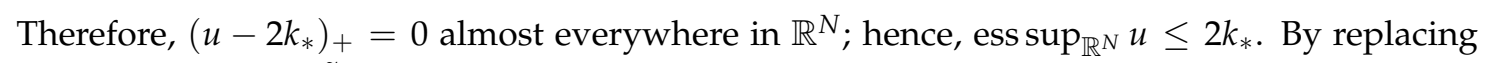
$u$ with $-u$ and $A_{k}$ with $\tilde{A}_{k}$, we analogously conclude that $u$ is bounded from below. Therefore, we conclude that

$$
\|u\|_{L^{\infty}\left(\mathbb{R}^{N}\right)} \leq \mathcal{C}\left(\int_{\mathbb{R}^{N}}|u|^{q} d x\right)^{\frac{\delta \tilde{q}}{q(\tilde{q}-q)}}
$$

This completes the proof.

We are ready to obtain our main result for the existence of a sequence of infinitely many small-energy solutions whose $L^{\infty}$ norms converge to zero. As mentioned above, the main tools are the modified functional method and the dual-fountain theorem. To do this, we assume that, for $p<q<p_{s}^{*}$,

(F3) For any $x \in \mathbb{R}^{N}$, there exists constant $s_{0}>0$, such that $p F(x, t)-f(x, t) t>0$ for $0<|t|<s_{0}$, where $F(x, t)=\int_{0}^{t} f(x, s) d s$.

(F4) $\lim _{|t| \rightarrow 0} \frac{f(x, t)}{|t|^{p-2} t}=+\infty$ uniformly for all $x \in \mathbb{R}^{N}$.

Remark 1. Define cut-off function $\kappa \in C^{1}(\mathbb{R}, \mathbb{R})$ satisfying $\kappa(t)=1$ for $|t| \leq t_{0}, \kappa(t)=0$ for $|t| \geq 2 t_{0}$, $\left|\kappa^{\prime}(t)\right| \leq 2 / t_{0}$, and $\kappa^{\prime}(t) t \leq 0$. Thus, we set

$$
\tilde{F}(x, t)=\kappa(t) F(x, t)+\left.(1-\kappa(t)) \xi\right|^{p} \quad \text { and } \quad \tilde{f}(x, t)=\frac{\partial}{\partial t} \tilde{F}(x, t),
$$

where $\xi$ is a positive constant. Then, the following Lemma holds.

On the basis of the work in $[24,38]$, we obtain the two following lemmas.

Lemma 7. Let Assumptions (V), (F1)-(F3) hold. Then,

$$
I(u)=0=\left\langle I^{\prime}(u), u\right\rangle \quad \text { if and only if } u=0 .
$$

Lemma 8. Assume that Assumptions (F1)-(F4) hold. Then, there exist $t_{0}>0$ with $t_{0}<\min \left\{s_{0}, 1\right\} / 2$ and $\tilde{f} \in C^{1}\left(\mathbb{R}^{N} \times \mathbb{R}, \mathbb{R}\right)$, such that $\tilde{f}(x, t)$ is odd for $t, \tilde{\mathcal{F}}(x, t) \geq 0$, and

$$
\tilde{\mathcal{F}}(x, t)=0 \quad \text { iff } \quad t \equiv 0 \quad \text { or } \quad|t| \geq 2 t_{0},
$$

where $\tilde{\mathcal{F}}(x, t):=p \tilde{F}(x, t)-\tilde{f}(x, t) t$ and $\frac{\partial}{\partial t} \tilde{F}(x, t)=\tilde{f}(x, t)$.

The following definition can be found in [51].

Definition 2. Let $\mathcal{X}$ be a reflexive Banach space. $\Theta$ satisfies the $(P S)_{c}^{*}$-condition (with respect to $\mathcal{Y}_{n}$ ) for every $c \in \mathbb{R}$ if any sequence $\left\{v_{n}\right\}_{n \in \mathbb{N}} \subset \mathcal{X}$ for which $v_{n} \in \mathcal{Y}_{n}$, for any $n \in \mathbb{N}$,

$$
\Theta\left(v_{n}\right) \rightarrow c \text { and }\left\|\left(\Theta \mid \mathcal{Y}_{n}\right)^{\prime}\left(v_{n}\right)\right\|_{\mathcal{X}^{*}} \rightarrow 0 \text { as } n \rightarrow \infty,
$$

contains a subsequence converging to a critical point of $\mathcal{X}$. 
Proposition 2. (Dual-Fountain Theorem ([51], Theorem 3.18)) Assume that $\mathcal{X}$ is a reflexive Banach space, $I \in C^{1}(\mathcal{X}, \mathbb{R})$ is an even functional. If there exists $k_{0}>0$ such that, for each $k \geq k_{0}$, there is $\rho_{k}>\delta_{k}>0$, such that

(D1) $\inf \left\{I(v): v \in \mathcal{Z}_{k},\|v\|_{\mathcal{X}}=\rho_{k}\right\} \geq 0$.

(D2) $\quad b_{k}:=\max \left\{I(v): v \in \mathcal{Y}_{k},\|v\|_{\mathcal{X}}=\delta_{k}\right\}<0$.

(D3) $d_{k}:=\inf \left\{I(v): v \in \mathcal{Z}_{k},\|v\|_{\mathcal{X}} \leq \rho_{k}\right\} \rightarrow 0$ as $k \rightarrow \infty$

(D4) I satisfies the $(P S)_{c}^{*}$-condition for every $c \in\left[d_{k_{0}}, 0\right)$,

then I has a sequence of negative critical values converging to 0 .

Theorem 1. Suppose that Assumptions (F1)-(F4) hold. If $f(x, t)$ is odd in $t$ for a small $t$, then the problem in Equation (1) admits a sequence of weak solutions $\left\{u_{n}\right\}$ satisfying $\left\|u_{n}\right\|_{L^{\infty}\left(\mathbb{R}^{N}\right)} \rightarrow 0$ as $n \rightarrow \infty$ for every

$$
\lambda \in \Gamma:=\left(0, \min \left(\frac{q}{p(\xi q \mathcal{C}+1)}, \frac{1}{p\left(2 \mathcal{C}\|\rho\|_{L^{p^{\prime}\left(\mathbb{R}^{N}\right)}}+\frac{\|\rho\|_{L^{\infty}\left(\mathbb{R}^{N}\right)}}{q}+\xi\right)}\right)\right) .
$$

Remark 2. As mentioned in the Introduction, even if the dual-fountain theorem plays a decisive role in obtaining the existence of multiple small-energy solutions to elliptic equations of the variational type, the boundedness of these solutions is not ensured by this theorem. The condition on nonlinear term $f(\cdot, t)$ at infinity, and the oddness of $f$ globally is essential in applying this theorem. However, by employing the dual-fountain theorem, we design our main theorem when $f(\cdot, t)$ is carried out near zero, and $f(\cdot, t)$ is odd in $t$ for a small $t$. Therefore, our approach for obtaining this consequence is somewhat different from former related works [8,9,24,29,38,41-44].

Proof. Consider modified energy functional $\widetilde{I}_{\lambda}: X\left(\mathbb{R}^{N}\right) \rightarrow \mathbb{R}$ given by

$$
\widetilde{I}_{\lambda}(v):=\Phi_{s, p}(v)-\lambda \widetilde{\Psi}(v),
$$

where $\Phi_{s, p}$ is given in Equation (2) and

$$
\widetilde{\Psi}(v)=\int_{\Omega} \widetilde{F}(x, v) d x, \quad v \in X\left(\mathbb{R}^{N}\right) .
$$

Then, it is clear by Lemma 8 that $\widetilde{I}_{\lambda} \in C^{1}\left(X\left(\mathbb{R}^{N}\right), \mathbb{R}\right)$ is an even functional. Now, we show that Conditions (D1)-(D4) of Proposition 2 are satisfied.

(D1) From Assumption (F2), we have

$$
|F(x, t)| \leq \rho(x)|t|+\frac{1}{q} \sigma(x)|t|^{q},(x, t) \in \mathbb{R}^{N} \times \mathbb{R} .
$$


For convenience, we denote $\theta_{k}=\sup _{\|u\|_{X\left(\mathbb{R}^{N}\right)}=1, u \in \mathcal{Z}_{k}}\|v\|_{L^{q}\left(\mathbb{R}^{N}\right)}$. It follows from the definition of $\kappa$ that we have

$$
\begin{aligned}
\widetilde{I}_{\lambda}(u): & =\frac{1}{p} \int_{\mathbb{R}^{N}} \int_{\mathbb{R}^{N}}|u(x)-u(y)|^{p} \mathcal{K}(x-y) d x d y+\frac{1}{p} \int_{\mathbb{R}^{N}} V(x)|u(x)|^{p} d x \\
& -\lambda \int_{\mathbb{R}^{N}} \kappa(u) F(x, u)+(1-\kappa(u)) \xi|u|^{p} d x \\
\geq & \frac{1}{p}\|u\|_{X\left(\mathbb{R}^{N}\right)}^{p}-\lambda \int_{\mathbb{R}^{N}} \rho(x)|u|+\frac{1}{q} \sigma(x)|u|^{q}+\xi|u|^{p} d x \\
\geq & \frac{1}{p}\|u\|_{X\left(\mathbb{R}^{N}\right)}^{p}-\lambda\|\rho\|_{L^{q^{\prime}}\left(\mathbb{R}^{N}\right)}\|u\|_{L^{q}\left(\mathbb{R}^{N}\right)}-\frac{\lambda}{q}\|\sigma\|_{L^{\infty}\left(\mathbb{R}^{N}\right)}\|u\|_{L^{q}\left(\mathbb{R}^{N}\right)}^{q}-\lambda \xi\|u\|_{L^{p}\left(\mathbb{R}^{N}\right)}^{p} \\
\geq & \frac{1}{p}\|u\|_{X\left(\mathbb{R}^{N}\right)}^{p}-\lambda \mathcal{C}\|u\|_{X\left(\mathbb{R}^{N}\right)}-\frac{\lambda}{q} \mathcal{C}\|u\|_{L^{q}\left(\mathbb{R}^{N}\right)}^{q}-\lambda \xi \|_{L^{p}\left(\mathbb{R}^{N}\right)}^{p} \\
\geq & \left(\frac{1}{p}-\lambda \xi \mathcal{C}\right)\|u\|_{X\left(\mathbb{R}^{N}\right)}^{p}-\lambda \mathcal{C}\|u\|_{X\left(\mathbb{R}^{N}\right)}-\frac{\lambda}{q} \mathcal{C} \theta_{k}^{q}\|u\|_{X\left(\mathbb{R}^{N}\right)}^{q} .
\end{aligned}
$$

Choose $\rho_{k}=\left(\mathcal{C} \theta_{k}^{q}\right)^{1 /(p-q)}$. Let $u \in \mathcal{Z}_{k}$ with $\|u\|_{X\left(\mathbb{R}^{N}\right)}=\rho_{k}>1$ for a sufficiently large $k$. Then, there exists $k_{0} \in \mathbb{N}$, such that

$$
\widetilde{I}_{\lambda}(u) \geq\left(\frac{1}{p}-\lambda \xi \mathcal{C}-\frac{\lambda}{q}\right) \rho_{k}^{p}-\lambda \mathcal{C} \rho_{k} \geq 0
$$

for all $k \in \mathbb{N}$ with $k \geq k_{0}$, because

$$
\lim _{k \rightarrow \infty}\left(\frac{1}{p}-\lambda \xi \mathcal{C}-\frac{\lambda}{q}\right) \rho_{k}^{p}=\infty
$$

Therefore,

$$
\inf \left\{\widetilde{I}_{\lambda}(u): u \in \mathcal{Z}_{k},\|u\|_{X\left(\mathbb{R}^{N}\right)}=\rho_{k}\right\} \geq 0 .
$$

(D2) $\|\cdot\|_{L^{\infty}\left(\mathbb{R}^{N}\right)},\|\cdot\|_{L^{p}\left(\mathbb{R}^{N}\right)}$ and $\|\cdot\|_{X\left(\mathbb{R}^{N}\right)}$ are equivalent on $\mathcal{Y}_{k}$. Then, there are positive constants $\kappa_{1}$ and $\kappa_{2}$ such that

$$
\kappa_{1}\|\cdot\|_{L^{\infty}\left(\mathbb{R}^{N}\right)} \leq\|\cdot\|_{X\left(\mathbb{R}^{N}\right)} \leq \kappa_{2}\|\cdot\|_{L^{p}\left(\mathbb{R}^{N}\right)}
$$

for any $v \in \mathcal{Y}_{k}$. From Assumptions (F3) and (F4), for any $\mathfrak{M}>0$, there exist $s_{1} \in\left(0, s_{0}\right)$ such that

$$
F(x, t) \geq \frac{\mathfrak{M} \kappa_{2}^{p}}{p}|t|^{p}
$$

for almost all $x \in \mathbb{R}^{N}$ and all $|t| \leq s_{1}$. Choose $\delta_{k}:=\min \left\{\frac{1}{2}, s_{1} \kappa_{1}\right\}$ for all $k \in \mathbb{N}$. Then, we know that $\|u\|_{L^{\infty}\left(\mathbb{R}^{N}\right)} \leq s_{1}$ for $u \in \mathcal{Y}_{k}$ with $\|u\|_{X\left(\mathbb{R}^{N}\right)}=\delta_{k}$, and thus $\tilde{F}(x, u)=F(x, u)$ for $\|u\|_{L^{\infty}\left(\mathbb{R}^{N}\right)} \leq s_{1}$. Hence, we derive by Equation (12) that

$$
\widetilde{I}_{\lambda}(u) \leq \frac{1}{p}\|u\|_{X\left(\mathbb{R}^{N}\right)}^{p}-\frac{\lambda \mathfrak{M} \kappa_{2}^{p}}{p}\|u\|_{L^{p}\left(\mathbb{R}^{N}\right)}^{p} \leq-\frac{\lambda \mathfrak{M}-1}{p}\|u\|_{X\left(\mathbb{R}^{N}\right)}^{p}=-\frac{(\lambda \mathfrak{M}-1) \delta_{k}^{p}}{p}<0
$$

for sufficiently large $\mathfrak{M}$ and for all $u \in \mathcal{Y}_{k}$ with $\|u\|_{X\left(\mathbb{R}^{N}\right)}=\delta_{k}$. We therefore obtain that

$$
b_{k}=\max \left\{\widetilde{I}_{\lambda}(u): u \in \mathcal{Y}_{k},\|u\|_{X\left(\mathbb{R}^{N}\right)}=\delta_{k}\right\}<0
$$

for all $k \in \mathbb{N}$, as claimed.

(D3) Because $\mathcal{Y}_{k} \cap \mathcal{Z}_{k} \neq \phi$ and $0<\delta_{k}<\rho_{k}$, we have $d_{k} \leq b_{k}<0$ for all $k \geq k_{0}$. Let us denote

$$
\widetilde{\theta}_{k}=\sup \left\{\int_{\mathbb{R}^{N}} \rho(x)|u(x)| d x: u \in \mathcal{Z}_{k},\|u\|_{X\left(\mathbb{R}^{N}\right)} \leq 1\right\} .
$$


Then, it is easy to verify that $\widetilde{\theta}_{k} \rightarrow 0$ as $k \rightarrow \infty$ (see [36]). For any $u \in \mathcal{Z}_{k}$ with $\|u\|_{X\left(\mathbb{R}^{N}\right)}=1$ and $0<t<\rho_{k}$, we have

$$
\begin{aligned}
\widetilde{I}_{\lambda}(t u) & =\frac{1}{p}\|t u\|_{X\left(\mathbb{R}^{N}\right)}^{p}-\lambda \int_{\mathbb{R}^{N}} \kappa(t u) F(x, t u)+\left(1-\kappa(t u) \xi|t u|^{p} d x\right. \\
& \geq\left(\frac{1}{p}-\lambda \xi \mathcal{C}\right)\|t u\|_{X\left(\mathbb{R}^{N}\right)}^{p}-\lambda \int_{\mathbb{R}^{N}}\left(\rho(x)|t u|+\frac{\sigma(x)}{q}|t u|^{q}\right) d x \\
& \geq-\lambda \rho_{k}^{q} \frac{\|\sigma\|_{L^{\infty}\left(\mathbb{R}^{N}\right)}}{q} \int_{\mathbb{R}^{N}}|u|^{q} d x-\lambda \rho_{k} \int_{\mathbb{R}^{N}} \rho(x)|u| d x \\
& \geq-\lambda \rho_{k}^{q} \frac{\|\sigma\|_{L^{\infty}\left(\mathbb{R}^{N}\right)}}{q} \theta_{k}-\lambda \rho_{k} \widetilde{\theta}_{k} .
\end{aligned}
$$

Hence, we achieve

$$
d_{k} \geq-\lambda \rho_{k}^{q} \frac{\|\sigma\|_{L^{\infty}\left(\mathbb{R}^{N}\right)}}{q} \theta_{k}-\lambda \rho_{k} \widetilde{\theta}_{k}
$$

Because $\theta_{k} \rightarrow 0$ and $\widetilde{\theta}_{k} \rightarrow 0$ as $k \rightarrow \infty$, we conclude that $\lim _{k \rightarrow \infty} d_{k}=0$.

(D4) Let $u \in X\left(\mathbb{R}^{N}\right)$ and $\|u\|_{X\left(\mathbb{R}^{N}\right)} \geq 1$. We set $\Omega_{1}:=\left\{x \in \mathbb{R}^{N}:|u(x)| \leq t_{0}\right\}$, $\Omega_{2}:=\left\{x \in \mathbb{R}^{N}: t_{0} \leq|u(x)| \leq 2 t_{0}\right\}$, and $\Omega_{3}:=\left\{x \in \mathbb{R}^{N}: 2 t_{0} \leq|u(x)|\right\}$, where $t_{0}$ is given in Lemma 8. From the relation in Equation (1) and the conditions of $\alpha$, we have

$$
\begin{aligned}
\widetilde{I}_{\lambda}(u) & :=\frac{1}{p}\|u\|_{X\left(\mathbb{R}^{N}\right)}^{p}-\lambda \int_{\mathbb{R}^{N}} \tilde{F}(x, u) d x \\
& \geq \frac{1}{p}\|u\|_{X\left(\mathbb{R}^{N}\right)}^{p}-\lambda \int_{\Omega_{1}} F(x, u) d x-\lambda \int_{\Omega_{2}}\left\{\kappa(u) F(x, u)+(1-\kappa(u)) \xi|u|^{p}\right\} d x-\lambda \int_{\Omega_{3}} \xi|u|^{p} d x \\
& \geq \frac{1}{p}\|u\|_{X\left(\mathbb{R}^{N}\right)}^{p}-\lambda \int_{\Omega_{1} \cup \Omega_{2}} F(x, u) d x-\lambda \int_{\Omega_{2} \cup \Omega_{3}} \xi|u|^{p} d x \\
& \geq \frac{1}{p}\|u\|_{X\left(\mathbb{R}^{N}\right)}^{p}-\lambda \int_{\Omega_{1} \cup \Omega_{2}} \rho(x)|u| d x-\lambda \int_{\Omega_{1} \cup \Omega_{2}} \frac{\sigma(x)}{q}|u|^{q} d x-\lambda \int_{\Omega_{2} \cup \Omega_{3}} \xi|u|^{p} d x \\
& \geq \frac{1}{p}\|u\|_{X\left(\mathbb{R}^{N}\right)}^{p}-2 \lambda\|\rho\|_{L^{p^{\prime}}\left(\mathbb{R}^{N}\right)}\|u\|_{L^{p}\left(\mathbb{R}^{N}\right)}-\lambda\left(\frac{\|\sigma\|_{L^{\infty}\left(\mathbb{R}^{N}\right)}}{q}+\xi\right) \int_{\mathbb{R}^{N}}|u|^{p} d x \\
& \geq \frac{1}{p}\|u\|_{X\left(\mathbb{R}^{N}\right)}^{p}-\lambda\left(2 \mathcal{C}\|\rho\|_{L^{p^{\prime}\left(\mathbb{R}^{N}\right)}}+\frac{\|\sigma\|_{L^{\infty}\left(\mathbb{R}^{N}\right)}}{q}+\xi\right)\|u\|_{X\left(\mathbb{R}^{N}\right)}^{p} .
\end{aligned}
$$

Therefore, we deduce that, for any

$$
\lambda \in\left(0, \frac{1}{p\left(2 \mathcal{C}\|\rho\|_{L^{p^{\prime}}\left(\mathbb{R}^{N}\right)}+\frac{\|\sigma\|_{L^{\infty}\left(\mathbb{R}^{N}\right)}}{q}+\xi\right)}\right)
$$

$\widetilde{I}_{\lambda}$ is coercive, that is, $\widetilde{I}_{\lambda}(u) \rightarrow \infty$ as $\|u\|_{X\left(\mathbb{R}^{N}\right)} \rightarrow \infty$; thus, it is bounded from below on $X\left(\mathbb{R}^{N}\right)$.

By Lemma 5 and similar arguments as those of Theorem 3 in [31], we claim that functional $\widetilde{\Psi}^{\prime}: X\left(\mathbb{R}^{N}\right) \rightarrow X^{*}\left(\mathbb{R}^{N}\right)$, defined by

$$
\left\langle\widetilde{\Psi}^{\prime}(u), \varphi\right\rangle=\int_{\mathbb{R}^{N}} \widetilde{f}(x, u) \varphi d x \text { for any } \varphi \in X\left(\mathbb{R}^{N}\right),
$$

is compact in $X\left(\mathbb{R}^{N}\right)$. It also follows from Lemmas 2.2 and 2.3 in [50] that $\Phi_{s, p}^{\prime}$ is strictly monotone, coercive, and mapping of type $\left(S_{+}\right)$. By the Browder-Minty theorem, the inverse operator of $\Phi_{s, p}^{\prime}$ exists (see Theorem 26.A in [53]). Since $\Phi_{s, p}^{\prime}$ is of type $\left(S_{+}\right)$, it is obvious that it has a continuous inverse. From the compactness of the derivative of $\widetilde{\Psi}$ and the coercivity of $\widetilde{I}_{\lambda}$, it follows that functional 
$\widetilde{I}_{\lambda}$ satisfies Condition $(P S)$. Because $X\left(\mathbb{R}^{N}\right)$ is a reflexive Banach space, the proof is carried out by the same scheme as that in ([36], Lemma 3.12).

Consequently, all conditions of Proposition 2 are satisfied; hence, for $\lambda \in \Gamma$, we have sequence $c_{n}<0$ for $\tilde{I}_{\lambda}$ satisfying $c_{n} \rightarrow 0$ when $n$ goes to $\infty$. Then, for any $u_{n} \in X\left(\mathbb{R}^{N}\right)$ satisfying $\tilde{I}_{\lambda}\left(u_{n}\right)=c_{n}$ and $\tilde{I}_{\lambda}^{\prime}\left(u_{n}\right)=0$, the sequence $\left\{u_{n}\right\}$ is a $(P S)$ sequence of $\tilde{I}_{\lambda}(u)$, and $\left\{u_{n}\right\}$ has a convergent subsequence. Thus, up to a subsequence, still denoted by $\left\{u_{n}\right\}$, one has $u_{n} \rightarrow u$ in $X\left(\mathbb{R}^{N}\right)$ as $n \rightarrow \infty$. Lemmas 7 and 8 imply that 0 is the only critical point with 0 energy, and subsequence $\left\{u_{n}\right\}$ has to converge to 0 in $X\left(\mathbb{R}^{N}\right)$; thus, $\left\|u_{n}\right\|_{L^{r}\left(\mathbb{R}^{N}\right)} \rightarrow 0$ as $n \rightarrow \infty$ for any $r$ with $p<r<p_{s}^{*}$. Owing to Proposition 1 , any weak solution $u$ of our problem belongs to space $L^{\infty}\left(\mathbb{R}^{N}\right)$, and there exist positive constants $C, \tau$ independent of $u$, such that

$$
\|u\|_{L^{\infty}\left(\mathbb{R}^{N}\right)} \leq C\|u\|_{L^{q}\left(\mathbb{R}^{N}\right)}^{\tau} .
$$

From this fact, we know $\left\|u_{n}\right\|_{L^{\infty}\left(\mathbb{R}^{N}\right)} \rightarrow 0$; thus, by Lemma 8 again, we have $\left\|u_{n}\right\|_{L^{\infty}\left(\mathbb{R}^{N}\right)} \leq s_{2}$ for a large $n$. Thus, $\left\{u_{n}\right\}$ with a large enough $n$ is a sequence of weak solutions of the problem in Equation (1). The proof is complete.

\section{Conclusions}

This paper is devoted to the study of the existence, multiplicity, and uniform estimates of infinitely many nontrivial weak solutions for nonlocal integrodifferential equations. The fountain and dual-fountain theorems as key tools are powerful techniques for ensuring the existence of multiple solutions to elliptic equations of the variational type. Subsequently, these theorems have been widely applied by many researchers to obtain the existence of multiple solutions for various problems of the elliptic type. The dual-fountain theorem is essential in deriving the existence of infinitely many small-energy solutions for nonlinear elliptic equations of the variational type. However, the boundedness of solutions cannot be obtained from this variational method. This theorem is also not applicable if we drop the condition on nonlinear term $f(\cdot, t)$ at infinity and the oddness of $f$ globally. To overcome this difficulty, the authors of $[24,38,41-44]$ employed the modified functional method and global variational formulation. In this regard, our attempt is new because we utilize the dual-fountain theorem in place of global variational formulation to obtain our main result.

Author Contributions: All authors (J.I.L., Y.-H.K., and J.L.) contributed equally to the writing of this paper. All authors have read and agreed to the published version of the manuscript.

Funding: This research received no external funding.

Acknowledgments: The authors gratefully thank to the referee for the constructive comments and recommendations that definitely helped to improve the readability and quality of the paper.

Conflicts of Interest: The authors declare no conflict of interest.

\section{References}

1. Bertoin, J. Levy Processes. Cambridge Tracts in Mathematics; Cambridge University Press: Cambridge, UK, 1996.

2. Bjorland, C.; Caffarelli, L.; Figalli, A. A Non-local gradient dependent operators. Adv. Math. 2012, 230, 1859-1894. [CrossRef]

3. Caffarelli, L. Nonlocal equations, drifts and games. In Nonlinear Partial Differential Equations, Abel Symposia; Springe: New York, NY, USA, 2012; Volume 7, pp. 37-52.

4. Gilboa, G.; Osher, S. Nonlocal operators with applications to image processing. Multiscale Model. Simul. 2008, 7, 1005-1028. [CrossRef]

5. Laskin, N. Fractional quantum mechanics and Levy path integrals. Phys. Lett. A 2000, 268, $298-305$. [CrossRef]

6. Metzler, R.; Klafter, J. The random walk's guide to anomalous diffusion: A fractional dynamics approach. Phys. Rep. 2003, 339, 1-77. [CrossRef]

7. Metzler, R.; Klafter, J. The restaurant at the random walk: Recent developments in the description of anomalous transport by fractional dynamics. J. Phys. A 2004, 37, 161-208. [CrossRef] 
8. Ge, B. Multiple solutions of nonlinear schrödinger equation with the fractional Laplacian. Nonlinear Anal. Real World Appl. 2016, 30, 236-247. [CrossRef]

9. Teng, K. Multiple solutions for a class of fractional Schrödinger equations in $\mathbb{R}^{N}$. Nonlinear Anal. Real World Appl. 2015, 21, 76-86. [CrossRef]

10. Zang, H.; Xu, J.; Zhang, F. Existence and multiplicity of solutions for superlinear fractional schrödinger equations in $\mathbb{R}^{N}$. J. Math. Phys. 2015, 56,1-13.

11. Autuori, G.; Pucci, P. Elliptic problems involving the fractional Laplacian in $\mathbb{R}^{N}$. J. Differ. Equ. 2013, 255, 2340-2362. [CrossRef]

12. Barrios, B.; Colorado, E.; de Pablo, A.; Sanchez, U. On some critical problems for the fractional Laplacian operator. J. Differ. Equ. 2012, 252, 6133-6162. [CrossRef]

13. Brasco, L.; Parini, E.; Squassina, M. Stability of variational eigenvalues for the fractional $p$-Laplacian. Discrete Contin. Dyn. Syst. 2016, 36, 1813-1845. [CrossRef]

14. Figueiredo, G.; Siciliano, G.A. Multiplicity result via Ljusternick-Schnirelmann category and Morse theory for a fractional schrödinger equation in $\mathbb{R}^{N}$. Nonlinear Differ. Equ. Appl. 2016, 23, 12. [CrossRef]

15. Iannizzotto, A.; Liu, S.; Perera, K.; Squassina, M. Existence results for fractional $p$-Laplacian problems via Morse theory. Adv. Calc. Var. 2016, 9, 101-125. [CrossRef]

16. Lehrer, R.; Maia, L.A.; Squassina, M. On fractional p-Laplacian problems with weight. Differ. Integral Equ. 2015, 28, 15-28.

17. Perera, K.; Squassina, M.; Yang, Y. Bifurcation and multiplicity results for critical fractional $p$-Laplacian problems. Math. Nachr. 2016, 289, 332-342. [CrossRef]

18. Servadei, R. Infinitely many solutions for fractional Laplace equations with subcritical nonlinearity. Contemp. Math. 2013, 595, 317-340.

19. Xu, J.; Wei, Z.; Dong, W. Existence of weak solutions for a fractional Schrödinger equation. Commun. Nonlinear Sci. Numer. Simul. 2015, 22, 1215-1222. [CrossRef]

20. Caliciotti, A.; Fasano, G.; Nash, S.G.; Roma, M. An adaptive truncation criterion, for linesearch-based truncated Newton methods in large scale nonconvex optimization. Oper. Res. Lett. 2018, 46, 7-12. [CrossRef]

21. Caliciotti, A.; Fasano, G.; Nash, S.G.; Roma, M. Data and performance profiles applying an adaptive truncation criterion, within linesearch-based truncated Newton methods, in large scale nonconvex optimization. Data Brief. 2018, 17, 246-255. [CrossRef]

22. Vergara, V.; Zacher, R. A priori bounds for degenerate and singular evolutionary partial integro-differential equations. Nonlinear Anal. 2010, 73, 3572-3585. [CrossRef]

23. Winkert, P.; Zacher, R. A priori bounds for weak solutions to elliptic equations with nonstandard growth. Discrete Contin. Dyn. Syst. Ser. S 2012, 5, 865-878.

24. Choi, E.B.; Kim, J.-M.; Kim, Y.-H. Infinitely many solutions for equations of $p(x)$-Laplace type with the nonlinear Neumann boundary condition. Proc. R. Soc. Edinb. Sect. A 2018, 148, 1-31. [CrossRef]

25. Bartsch, T.; Willem, M. On an elliptic equation with concave and convex nonlinearities. Proc. Am. Math. Soc. 1995, 123, 3555-3561. [CrossRef]

26. Bartsch, T.; Wang, Z.-Q. Existence and multiplicity results for some superlinear elliptic problems on $\mathbb{R}^{N}$. Comm. Partial Differ. Equ. 1995, 20, 1725-1741. [CrossRef]

27. Ambrosetti, A.; Rabinowitz, P. Dual variational methods in critical point theory and applications. J. Funct. Anal. 1973, 14, 349-381. [CrossRef]

28. Alves, C.O.; Liu, S.B. On superlinear $p(x)$-Laplacian equations in $\mathbb{R}^{N}$. Nonlinear Anal. 2010, 73, $2566-2579$. [CrossRef]

29. Dai, G.; Hao, R. Existence of solutions for a $p(x)$-Kirchhoff-type equation. J. Math. Anal. Appl. 2009, 359, 275-284 . [CrossRef]

30. Kim, J.-M.; Kim, Y.-H.; Lee, J. Existence and multiplicity of solutions for Kirchhoff-Schrödinger type equations involving $p(x)$-Laplacian on the whole space. Nonlinear Anal. Real World Appl. 2019, 45, 620-649.

31. Kim, J.-M.; Kim, Y.-H.; Lee, J. Multiplicity of small or large energy solutions for Kirchhoff-Schrödinger-type equations involving the fractional $p$-Laplacian in $\mathbb{R}^{N}$. Symmetry 2018, 10, 436. [CrossRef]

32. Lin, X.; Tang, X.H. Existence of infinitely many solutions for $p$-Laplacian equations in $\mathbb{R}^{N}$. Nonlinear Anal. 2013, 92, 72-81. [CrossRef]

33. Liu, S.B. On ground states of superlinear $p$-Laplacian equations in $\mathbb{R}^{N}$. J. Math. Anal. Appl. 2010, 361, 48-58. [CrossRef] 
34. Liu, S.B. On superlinear problems without Ambrosetti and Rabinowitz condition. Nonlinear Anal. 2010, 73, 788-795. [CrossRef]

35. Liu, S.B.; Li, S.J. Infinitely many solutions for a superlinear elliptic equation. Acta Math. Sin. (Chin. Ser.) 2003, 46, 625-630.

36. Miyagaki, O.H.; Hurtado, E.J.; Rodrigues, R.S. Existence and multiplicity of solutions for a class of elliptic equations without Ambrosetti-Rabinowitz type conditions. J. Dyn. Diff. Equ. 2018, 30, 405-432.

37. Zou, W. Variant fountain theorems and their applications. Manuscripta Math. 2001, 104, 343-358. [CrossRef]

38. Wang, Z.-Q. Nonlinear boundary value problems with concave nonlinearities near the origin. Nonlinear Differ. Equ. Appl. 2001, 8, 15-33. [CrossRef]

39. Heinz, H.P. Free Ljusternik-Schnirelman theory and the bifurcation diagrams of certain singular nonlinear problems. J. Differ. Equ. 1987, 66, 263-300. [CrossRef]

40. Dràbek, P.; Kufner, A.; Nicolosi, F. Quasilinear Elliptic Equations with Degenerations and Singularities; Walter de Gruyter \& Co.: Berlin, Germany, 1997.

41. Guo, Z. Elliptic equations with indefinite concave nonlinearities near the origin. J. Math. Anal. Appl. 2010, 367, 273-277. [CrossRef]

42. Kim, Y.-H. Infinitely many small energy solutions for equations involving the fractional Laplacian in $\mathbb{R}^{N}$. J. Korean Math. Soc. 2018, 55, 1269-1283.

43. Naimen, D. Existence of infinitely many solutions for nonlinear Neumann problems with indefinite coefficients. Electron. J. Differ. Equ. 2014, 2014, 181.

44. Tan, Z.; Fang, F. On superlinear $p(x)$-Laplacian problems without Ambrosetti and Rabinowitz condition. Nonlinear Anal. 2012, 75, 3902-3915. [CrossRef]

45. Adams, R.A.; Fournier, J.J.F. Sobolev Spaces, 2nd ed.; Academic Press: New York, NY, USA; London, UK, 2003.

46. Nezza, E.D.; Palatucci, G.; Valdinoci, E. Hitchhiker's guide to the fractional Sobolev spaces. Bull. Sci. Math. 2012, 136, 521-573. [CrossRef]

47. Xiang, M.; Zhang, B.; Ferrara, M. Existence of solutions for Kirchhoff type problem involving the non-local fractional p-Laplacian. J. Math. Anal. Appl. 2015, 424, 1021-1041. [CrossRef]

48. Demengel, F.; Demengel, G. Functional Spaces for the Theory of Elliptic Partial Differential Equations; Transl. from the 2007 French original by R. Ernè; Universitext; Springer: London, UK, 2012.

49. Perera, K.; Squassina, M.; Yang, Y. Critical fractional $p$-Laplacian problems with possibly vanishing potentials. J. Math. Anal. Appl. 2016, 433, 818-831. [CrossRef]

50. Torres Ledesma, C.E. Existence and symmetry result for fractional $p$-Laplacian in $\mathbb{R}^{n}$. Commun. Pure Appl. Anal. 2017, 16, 99-113. [CrossRef]

51. Willem, M. Minimax Theorems; Birkhauser: Basel, Switzerland, 1996.

52. Bonder, J.; Saintier, N.; Silva, A. The concentration-compactness principle for fractional order Sobolev spaces in unbounded domains and applications to the generalized fractional Brezis-Nirenberg problem. Nonlinear Differ. Equ. Appl. 2018, 25, 1-25. [CrossRef]

53. Zeidler, E. Nonlinear Functional Analysis and Its Applications. II/B: Nonlinear Monotone Operators; Springer: Berlin, Germany, 1990.

(c) 2019 by the authors. Licensee MDPI, Basel, Switzerland. This article is an open access article distributed under the terms and conditions of the Creative Commons Attribution (CC BY) license (http://creativecommons.org/licenses/by/4.0/). 